\title{
VINCULAÇÃO EM CASAIS: AVALIAÇÃO DA REPRESENTAÇÃO DA INTIMIDADE E DA INTERACÇÃO CONJUGAL
}

\author{
Vânia Lima ${ }^{1}$ \\ Filipa Vieira ${ }^{2}$ \\ Isabel Soares ${ }^{3}$
}

\begin{abstract}
Resumo: Tendo a Teoria da Vinculação de Bowlby como referencial para o estudo das relações íntimas na idade adulta, a investigação tem dedicado uma crescente atenção à especificidade da vinculação na idade adulta, no contexto das relações íntimas, podendo ser distinguidas, genericamente, duas linhas de investigação: uma linha focada no nível representacional e uma outra linha de natureza comportamental, focada em aspectos específicos da interaç̧ão conjugal. O presente artigo centra-se na relação entre a qualidade da representação da(s) relação(ões) íntima(s) e o comportamento nesse contexto relacional, pelo recurso à Intimate Relationship Interview (Lima, Vieira, Soares \& Collins, 2005) e à Couple Interaction Task (Collins, Hennighausen, Madsen \& Roisman, 1998). Resultados preliminares apontam para a associação entre a qualidade da organização da representação e comportamentos específicos de vinculação, salientando a pertinência da avaliação da vinculação na idade adulta, no contexto das relações íntimas, pelo recurso à combinação de múltiplos métodos.
\end{abstract}

Palavras-chave: vinculação na idade adulta; intimidade; interacção conjugal

Attachment in Couple's Relationship: Representation of Intimacy and Couple's Interaction (Abstract): Adopting Bowlby's attachment theory as the framework to the study of couples' relationships, there has been a growing interest of research regarding the specific contribution attachment plays in adult intimate relationships. Generally, two different research approaches may be distinguished: a representational and a behavioural one, focused on specific features of couple's interaction. This paper addresses the relation between the quality of representation of

\footnotetext{
${ }^{1}$ Bolseira de Doutoramento da Fundação de Ciência de Tecnologia (BDSFRH/BD/ 13291/2003). Informação sobre o artigo poderá ser solicitada através do e-mail vania.lima@iep.uminho.pt.

2 Assistente Estagiária da Faculdade de Psicologia e de Ciências da Educação da Universidade do Porto.

${ }^{3}$ Professora Catedrática do Departamento de Psicologia da Universidade do Minho.

PSICOLOGIA, Vol. XX (1), 2006, Edições Colibri, Lisboa, pp. 51-63
} 
the intimate relationship and the behaviour within this relational context, using the Intimate Relationship Interview (Lima, Vieira, Soares \& Collins, 2005) and the Couple Interaction Task (Collins, Hennighausen, Madsen \& Roisman, 1998). Preliminary data suggest a relation between the quality of representation of the intimate relationship and the specific attachment behaviours, emphasizing the relevance of combining multiple assessment methods when considering attachment in adult intimate relationships.

Key-words: adult attachment; intimacy; couple's interaction

\section{Introdução}

Adoptando como referencial teórico para o estudo das relações íntimas na idade adulta a Teoria da Vinculação de Bowlby (1969/1982), o presente artigo centra-se na qualidade da representação das relações íntimas e no comportamento nesse contexto relacional.

De acordo com a teoria de Bowlby, é desde a infância que podem ser encontrados os ingredientes para o estabelecimento de relações íntimas na adolescência e idade adulta. Este processo tem sido compreendido à luz do constructo de natureza cognitivo-emocional designado modelos internos dinâmicos de vinculação, concebidos como sendo construídos no contexto da relação da criança com as figuras de vinculação e que permitem organizar a experiência sob a forma de representações generalizadas sobre o self, as figuras de vinculação e as relações (Soares, 2000). Conforme sugerido por vários estudos de natureza longitudinal (e.g., Roisman, Madsen, Hennighausen, Sroufe \& Collins, 2001; Grossmann, Grossmann, Winter \& Zimmermann, 2002), os modelos internos dinâmicos são entendidos como relativamente estáveis ao longo do tempo, mas com possibilidade de reelaboração e de desenvolvimento em direcção a formas mais complexas e sofisticadas de organização da experiência e de orientação da acção, contribuindo para tal as experiências relacionais do indivíduo (Bowlby, 1988; Baldwin \& Fehr, 1985; Bretherton \& Munholland, 1999).

$\mathrm{Se}$ as relações precocemente estabelecidas entre a criança e a(s) sua(s) figura(s) de vinculação podem ser entendidas como prototípicas das relações íntimas na idade adulta (Waters, Kondo-Ikemura, Posada \& Richters, 1991; Roisman et al., 2001), a especificidade da vinculação no contexto das relações íntimas assenta na simetria e reciprocidade (Berman, Marcus \& Berman, 1994; West \& Sheldon-Keller, 1994; Crittenden, 1997; Crowell \& Treboux, 2001). Assim, de modo diferente do que ocorre na relação entre a criança e as suas figuras parentais, caracterizada pela complementaridade de papéis no que concerne à procura e prestação de cuida- 
dos, na relação adulto-adulto, é esperado um balanceamento contínuo e flexível entre o procurar e o proporcionar segurança e conforto, isto é, entre o recorrer ao outro como base segura e constituir-se como tal, face ao outro, quando necessário (Ainsworth, 1991; Crowell \& Treboux, 2001). Experienciar o outro como base segura, percebendo-o como disponível e responsivo face ao desconforto pessoal ou no confronto com situações percebidas como problemáticas, e, reciprocamente, funcionar como base segura para o outro confere ao indivíduo a percepção de segurança, pertença e partilha, consubstanciando o estabelecimento e desenvolvimento da intimidade (Cassidy, 2001).

No decurso da última década, os investigadores têm dedicado uma crescente atenção à especificidade da vinculação na idade adulta, no contexto de relações íntimas, podendo ser distinguidas, pelo menos, duas linhas de investigação: uma linha focada no nível representacional e uma outra linha, de natureza comportamental, focada em aspectos específicos da interacção conjugal. Relativamente à primeira linha, podemos distinguir dois tipos de avaliação centrada (1) na representação da vinculação com as figuras de vinculação na infância e ao longo do desenvolvimento e (2) na representação da relação de vinculação estabelecida pelo casal (Crowell \& Owens, 1998; Waters \& Cummings, 2000; Roisman, 2001).

A avaliação da representação da vinculação com as figuras de vinculação na infância e ao longo do desenvolvimento tem sido conduzida através da Adult Attachment Interview - AAI (George, Kaplan \& Main, 1985), uma entrevista de tipo clínico, semi-estruturada e de natureza biográfica, constituída por um conjunto de temas relevantes, tais como, por exemplo, separações, perdas, experiências de abandono, de rejeição e maus tratos. A avaliação desta entrevista valoriza a qualidade da organização da narrativa e o modo como o indivíduo integra as suas memórias no quadro mais geral das suas relações de vinculação. O sistema de cotação da entrevista assenta no relato e na descrição das experiências, na linguagem utilizada e no modo como o sujeito se revela capaz de integrar a diversidade das suas experiências e atribuir-lhes um significado coerente, conduzindo à identificação de três padrões ou organizações da vinculação - seguro/autónomo, inseguro/preocupado ou emaranhado, inseguro/desligado - e, ainda, à atribuição da categoria ou estatuto não resolvido/desorganizado.

Na sequência da $A A I$, mas orientada para a avaliação da representação das relações íntimas entre adultos, foi desenvolvida a Current Relationship Interview - CRI (Crowell, 1990). Nesta entrevista, onde o parceiro é assumido como uma figura de vinculação, o foco é colocado na avaliação da qualidade da representação da relação, do self e do outro na relação. À semelhança do que ocorre na $A A I$, valoriza-se a qualidade da organização da narrativa (nas dimensões semântica e episódica), permitin- 
do a classificação da entrevista de acordo com padrões de organização da vinculação equivalentes aos identificados na $A A I$.

No âmbito desta linha de investigação com a $C R I$, desenvolvemos a Intimate Relationship Interview - IRI (Lima, Soares, Vieira \& Collins, 2005), uma entrevista semi-estruturada destinada à avaliação de aspectos particulares da representação das relações íntimas. As questões da IRI permitem examinar o modo como o sujeito organiza internamente as suas experiências no contexto das relações íntimas, como as integra e lhes atribui significado e quais as principais estratégias comportamentais que utiliza. Tendo por base os contributos teóricos referidos anteriormente, a avaliação da $I R I$ considera dois níveis de análise: por um lado, o nível semântico, centrado no modo como o indivíduo descreve as suas experiências e as avaliações e interpretações que apresenta e, por outro lado, o nível episódico traduzido na capacidade do indivíduo sustentar as suas descrições, através de acontecimentos específicos e relevantes do ponto de vista do seu relacionamento íntimo.

No âmbito da segunda linha de investigação sobre a vinculação no contexto das relações íntimas, a observação da relação do casal assenta na realização de tarefas desafiantes, no sentido de avaliar comportamentos diádicos, atendendo, por um lado, a comportamentos de expressão e regulação do afecto, negociação e resolução de conflitos e, por outro lado, a comportamentos específicos de vinculação ao nível da existência de uma base segura, da prestação ou pedido de cuidados. Nestas tarefas, sugere-se ao casal a discussão de um aspecto problemático vivenciado no contexto da relação ou experienciado por um dos elementos fora do contexto da relação (Collins \& Feeney, 2000; Roisman et al., 2001; Crowell, Treboux, Gao, Fyffe, Pan \& Waters, 2002; Grossmann, Grossmann, Winter \& Zimmermann, 2002; Wampler, Shi, Nelson, Kimball, 2003), activando, deste modo, o sistema de vinculação, similarmente ao que ocorre com outros procedimentos, de natureza representacional, como a $A A I$ ou a $C R I$.

A integração destes diferentes métodos de avaliação - a entrevista e a observação da interacção - poderá permitir o desenvolvimento de leituras mais compreensivas e multifacetadas sobre a especificidade da vinculação na idade adulta, em relações íntimas, particularmente se atendermos à crescente evidência empírica de que a qualidade da representação da relação de vinculação com o companheiro está relacionada com a qualidade do comportamento observável no contexto de relações íntimas, quer ao nível de dimensões gerais, quer em dimensões específicas de vinculação (Roisman et al., 2001; Crowell et al., 2002).

Nesta linha de investigação, o nosso estudo tem como objectivo explorar as relações entre as dimensões específicas da vinculação, nos domínios representacional e comportamental. Em particular, são explorados 
índices gerais de organização da narrativa, como a coerência e a idealização e a forma como se relacionam com a representação do desenvolvimento pessoal, no contexto da relação, e a representação da prestação e procura de cuidados. No domínio comportamental, é avaliada a relação entre a qualidade global da relação e o comportamento de base segura e de desenvolvimento pessoal e relacional. Finalmente, são examinadas as relações entre o nível representacional e o nível comportamental da vinculação, no contexto da relação íntima.

\section{Método}

\section{Participantes}

A amostra é constituída por sete casais com idades compreendidas entre os 26 e os 34 anos de idade ( $\mathrm{M}=27,71$; $\mathrm{DP}=2,12)$. Em média, a duração dos relacionamentos é de 7 anos e 4 meses ( $D P=24,4$ meses), todos coabitam há, pelo menos, 2 anos e 6 meses $(\mathrm{M}=31,4$ meses; $\mathrm{DP}=24,4$ meses) e apenas um casal não é casado.

\section{Medidas e Procedimento}

\section{Avaliação da Representação da Relação Íntima}

A Intimate Relationship Interview - IRI (Lima, Soares, Vieira \& Collins, 2005) é uma entrevista semi-estruturada desenvolvida para avaliar a representação da relação íntima por parte de cada um dos elementos do casal. O protocolo da entrevista incide em cinco domínios relevantes para a compreensão da especificidade das relações íntimas, nomeadamente sobre a regulação emocional, intimidade sexual, capacidade de procurar e de proporcionar cuidados de base segura.

A primeira questão da IRI incide na influência, ao nível da relação íntima actual, de outras relações do passado, nomeadamente com as figuras de vinculação na infância e ao longo do desenvolvimento. Em seguida, um outro conjunto de questões explora os padrões de interacção relacionados com a procura e prestação de cuidados, quer em situações de desconforto não directamente relacionadas com a relação, tais como problemas no contexto laboral ou com amigos, quer em situações associadas com a própria relação, como um conflito ou desentendimento do casal. Na sequência destas questões, outras incidem na regulação de proximidade e distância face ao outro, bem como na procura de proximidade, contacto físico e envolvimento sexual com o outro, ao nível da comunicação, satisfação e expressão do desejo. A IRI foca, por último, a satisfação, expectativas e 
aprendizagens associadas à relação, procurando avaliar em que medida o indivíduo considera a sua relação como promotora de crescimento pessoal, num balanceamento entre as necessidades individuais e da relação.

A IRI é gravada em registo áudio, transcrita e cotada nas seguintes dimensões: integração de emocionalidade negativa e positiva, careseeking, caregiving, intimidade sexual, equilíbrio entre o desenvolvimento do indivíduo e o desenvolvimento da relação, idealização e coerência.

A escala de Integração da Emocionalidade Negativa e Positiva centra-se na avaliação da expressão de afecto negativo (como verbalizações que reflectem frustração, raiva ou hostilidade) e de afecto positivo (e.g.: expressão de sentimentos positivos dirigidos ao outro) e na sua integração num todo organizado e coerente. A escala de careseeking avalia a capacidade do sujeito procurar cuidados e de aceitar ser confortado pelo outro. Esta escala atende também à capacidade do indivíduo sinalizar ao outro o seu mal-estar, expressando a sua vulnerabilidade de forma clara e a disponibilidade a ser ajudado, mantendo estes sinais até que o outro os reconheça e o conforte. Complementarmente, a escala de caregiving avalia a capacidade do indivíduo reconhecer os sinais de vulnerabilidade e de mal-estar do outro, disponibilizando-se a lhe prestar cuidados e a satisfazer as suas necessidades de conforto e protecção, assumindo-se, assim, como base segura para o outro. A escala de Intimidade Sexual incide na proximidade física e emocional, na abertura e à-vontade no contexto da expressão sexual, pela capacidade do indivíduo comunicar com o outro sobre tópicos de natureza sexual. A última escala de avaliação específica da relação é sobre o Desenvolvimento do Indivíduo versus o Desenvolvimento da Relação procurando examinar em que medida o sujeito é capaz de, no contexto da relação, experienciar crescimento pessoal e percepcionar-se como autónomo, ou, se pelo contrário, a relação constrange os seus recursos ou inibe as necessidades e objectivos individuais. Para a avaliação da qualidade da organização da experiência, foram desenvolvidas duas escalas: a idealização avalia a discrepância entre a percepção geral que o indivíduo apresenta de si próprio, do outro, de si na relação e da relação per se, e os episódios que descreve sobre esses aspectos; a escala da coerência atende à precisão e clareza do discurso do indivíduo relativamente ao outro, a si e à relação, e à capacidade de se manter centrado nos tópicos da entrevista, apresentar evidência para as suas avaliações e percepções gerais, reflectir e integrar as suas experiências no contexto da relação.

Cada $I R I$ foi cotada por grupos de três juízes, de modo independente, previamente formados no método de avaliação, tendo sido obtido um acordo inter-juízes entre 0,80 e 0,93. 


\section{Avaliação do Comportamento na Interacção Diádica}

A Couple Interaction Task - CIT (Collins, Hennighausen, Madsen \& Roisman, 1998) é uma tarefa que envolve dois momentos distintos. Num primeiro momento, o casal deve seleccionar numa lista de possíveis problemas na relação um aspecto que seja suscitador de discórdia na díade. É, então, solicitado ao casal que converse sobre o problema e procure resolver o conflito, de modo a alcançar uma solução satisfatória para ambos. Num segundo momento, é solicitado ao casal que coopere na descrição do que para si seria uma relação ideal ou um casal perfeito (devendo assim descentrar-se da própria relação), por recurso à metodologia $Q$-sort.

A CIT é gravada em registo de vídeo e cotada através de um conjunto de escalas organizadas em cinco dimensões: tonalidade emocional, processo, equilíbrio/balanceamento, avaliação global e cuidados. Na dimensão de tonalidade emocional, surgem as escalas de Partilha de Afecto Positivo, Partilha de Afecto Negativo, Raiva e Hostilidade. A escala Partilha de Afecto Positivo é dirigida à avaliação da reciprocidade da expressão de afecto positivo pelo casal, centrando-se no grau de sincronia/sintonia e no reconhecimento de afecto positivo entre os elementos da díade. A escala Partilha de Afecto Negativo avalia a reciprocidade da expressão de afecto negativo pelo casal, ao nível da manifestação de frustração, impaciência, raiva e/ou hostilidade na interacção. Na escala de Raiva, é avaliada a intensidade de expressão de frustração ou impaciência dirigida ao parceiro (e não a terceiros ou à tarefa), ao passo que a Hostilidade reporta-se a interacções marcadas pela frieza emocional e rejeição.

Para as dimensões de processo há duas escalas: a Resolução de Conflito incide na capacidade do casal trabalhar em conjunto para a tomada de decisões ou resolução de conflito, conduzindo à mútua satisfação; a escala Base Segura avalia o modo como, no contexto da relação, os indivíduos são capazes de recorrer ao outro como base segura e de se constituírem como base segura para o parceiro.

As escalas que constituem a dimensão equilíbrio/balanceamento são o Equilíbrio entre Assertividade e Self-Concealment, Equilíbrio entre Desenvolvimento do Indivíduo e Desenvolvimento da Relação, Equilibrio entre Relação e Mundo. A escala de Equilíbrio entre Assertividade e Self-Concealment centra-se em aspectos como abertura e capacidade de exprimir ideias e opiniões de modo assertivo versus passividade e/ou defensividade na expressão de ideias ou opiniões, de forma a não ameaçar o outro ou a relação. A escala de Equilíbrio entre Desenvolvimento do Indivíduo e Desenvolvimento da Relação avalia em que medida a relação nutre/promove os parceiros como indivíduos, ou, ao invés, constrange os seus recursos individuais. A última escala da dimensão de balanceamento é a de Equilíbrio entre Relação e Mundo, que se orienta para a capacidade da díade manter um en- 
volvimento activo, uma dinâmica "interna" competente no "trabalhar em conjunto" dirigida ao cumprimento das instruções e da tarefa.

A Avaliação Global da Qualidade da Relação constitui uma avaliação holística da qualidade e profundidade da relação, não derivando de um compósito dos scores anteriores.

Para além destas dimensões de avaliação propostas pelos autores, introduzimos a dimensão de Cuidados, que comporta as escalas de avaliação individual de Careseeking e Caregiving. A escala Careseeking incide na capacidade do indivíduo recorrer ao parceiro, sinalizando o seu mal-estar e exprimindo disponibilidade para ser por ele confortado. Complementarmente, a escala de Caregiving avalia a capacidade do sujeito se mostrar disponível ao outro, reconhecendo o seu desconforto e sendo-lhe responsivo e eficaz na prestação de cuidados.

Cada CIT é cotada por grupos de três juízes, de modo independente, previamente formados no método de avaliação, tendo sido obtido um acordo inter-juízes entre 0,96 e 0,99 .

Os casais foram previamente contactados e informados relativamente ao estudo por um dos elementos do Grupo de Estudos de Vinculação. Após obtenção do seu consentimento informado, a avaliação realizou-se no laboratório ou em suas casas, realizando-se a IRI individualmente e antes da CIT.

\section{Resultados}

No Quadro 1, são apresentadas as associações entre as dimensões específicas da vinculação no domínio representacional, verificando-se que a Idealização está negativamente correlacionada com a Coerência $\left(\mathrm{r}_{\mathrm{sp}}=-0,694 ; \mathrm{p}<0,01\right)$, Caregiving $\left(\mathrm{r}_{\mathrm{sp}}=-0,763 ; \mathrm{p}<0,01\right)$ e Desenvolvimento do Indivíduo/Desenvolvimento da Relação $\left(\mathrm{r}_{\mathrm{sp}}=-0,616 ; \mathrm{p}<0,05\right)$.

Quadro 1: Correlações entre as escalas da IRI

\begin{tabular}{lllllc}
\hline & \multicolumn{1}{c}{$\begin{array}{c}\text { Ideali- } \\
\text { zação }\end{array}$} & $\begin{array}{c}\text { Coerên- } \\
\text { cia }\end{array}$ & $\begin{array}{c}\text { Des. Indi- } \\
\text { víduo/ Des. } \\
\text { Relação }\end{array}$ & $\begin{array}{c}\text { Care- } \\
\text { seeking }\end{array}$ & $\begin{array}{c}\text { Care- } \\
\text { giving }\end{array}$ \\
\hline $\begin{array}{l}\text { Idealização } \\
\text { Coerência }\end{array}$ & $-0,694^{* *}$ & - & - & - & - \\
$\begin{array}{l}\text { Des. Indivíduo/ } \\
\text { Des. Relação }\end{array}$ & $-0,616^{*}$ & $0,625^{*}$ & 1 & - & - \\
$\begin{array}{l}\text { Careseeking } \\
\text { Caregiving }\end{array}$ & $-0,499$ & 0,236 & $0,701^{* *}$ & 1 & - \\
\hline
\end{tabular}

Nota: $* \mathrm{p}<0,05 ; * * \mathrm{p}<0,01$. 
Correlações positivas são encontradas entre a escala de Desenvolvimento do Individuo/Desenvolvimento da Relação e as de Coerência $\left(\mathrm{r}_{\mathrm{sp}}=0,625\right.$; $\mathrm{p}<0,05)$ e Careseeking $\left(\mathrm{r}_{\mathrm{sp}}=0,701 ; \mathrm{p}<0,01\right)$. No âmbito dos Cuidados verifica-se uma correlação positiva entre as dimensões de Caregiving e Careseeking $\left(\mathrm{r}_{\mathrm{sp}}=0,737 ; \mathrm{p}<0,01\right)$.

No Quadro 2, relativo à dimensão comportamental, podem ser observadas as associações positivas entre a escala Qualidade da Relação e as escalas Desenvolvimento do Indivíduo/Desenvolvimento da Relação $\left(\mathrm{r}_{\mathrm{sp}}=0,889 ; \mathrm{p}<0,01\right)$ e Base Segura $\left(\mathrm{r}_{\mathrm{sp}}=0,904 ; \mathrm{p}<0,01\right)$. De igual modo, verifica-se uma associação positiva entre Base Segura e Desenvolvimento do Indivíduo/Desenvolvimento da Relação $\left(\mathrm{r}_{\mathrm{sp}}=0,889 ; \mathrm{p}<0,01\right)$.

Quadro 2: Correlações entre as escalas da CIT

\begin{tabular}{lccc}
\hline & $\begin{array}{c}\text { Qualidade da } \\
\text { Relação }\end{array}$ & $\begin{array}{c}\text { Des. Indivíduo/ } \\
\text { Des. Relação }\end{array}$ & $\begin{array}{c}\text { Base } \\
\text { Segura }\end{array}$ \\
\hline $\begin{array}{l}\text { Qualidade da Relação } \\
\text { Des. Indivíduo/ }\end{array}$ & 1 & - & - \\
$\begin{array}{l}\text { Des. Relação } \\
\text { Base Segura }\end{array}$ & $0,889 * *$ & 1 & - \\
\hline
\end{tabular}

Nota: ${ }^{*} \mathrm{p}<0,01$.

Quadro 3: Correlações entre as escalas da IRI e da CIT

\begin{tabular}{|c|c|c|c|c|c|}
\hline & $\begin{array}{c}\text { IRI } \\
\text { Ideali- } \\
\text { zação }\end{array}$ & $\begin{array}{c}\text { IRI } \\
\text { Coe- } \\
\text { rência }\end{array}$ & $\begin{array}{c}\text { CIT } \\
\text { Qualidade da } \\
\text { Relação }\end{array}$ & $\begin{array}{c}\text { CIT } \\
\text { Des. Indivíduo } \\
\text { /Des. Relação }\end{array}$ & $\begin{array}{c}\text { CIT } \\
\text { Base } \\
\text { Segura } \\
\end{array}$ \\
\hline $\begin{array}{c}\text { IRI } \\
\text { Idealização }\end{array}$ & 1 & - & - & - & - \\
\hline $\begin{array}{c}\text { IRI } \\
\text { Coerência } \\
\text { CIT }\end{array}$ & $-0,694 * *$ & 1 & - & - & - \\
\hline $\begin{array}{l}\text { Qualidade da } \\
\text { Relação } \\
\text { CIT }\end{array}$ & $-0,441$ & $0,694 * *$ & 1 & - & - \\
\hline $\begin{array}{l}\text { Des. Indivíduo } \\
\text { /Des. Relação }\end{array}$ & $-0,566^{*}$ & $0,749 * *$ & $0,889 * *$ & 1 & - \\
\hline $\begin{array}{c}\text { CIT } \\
\text { Base Segura }\end{array}$ & $-0,458$ & $0,722 * *$ & $0,904 * *$ & $0,939 * *$ & 1 \\
\hline
\end{tabular}

Nota: $* \mathrm{p}<0,05 ; *$ p $<0,01$ 
A análise das relações entre os níveis representacional e comportamental (Quadro 3) permite-nos destacar relações significativas entre a escala de Coerência e as escalas avaliadas ao nível comportamental relativas à Qualidade da Relação $\left(\mathrm{r}_{\mathrm{sp}}=0,694 ; \mathrm{p}<0,01\right)$, Base Segura $\left(\mathrm{r}_{\mathrm{sp}}=0,722\right.$; $\mathrm{p}<0,01)$ e Desenvolvimento do Indivíduo/Desenvolvimento da Relação $\left(\mathrm{r}_{\mathrm{sp}}=0,749 ; \mathrm{p}<0,01\right)$. Esta última escala encontra-se, ainda, inversamente correlacionada com a escala Idealização no domínio representacional $\left(r_{s p}=-0,566 ; p<0,05\right)$.

\section{Discussão}

Os resultados apresentados merecem a nossa reflexão do ponto de vista metodológico e do ponto de vista teórico.

Neste sentido, e atendendo especificamente aos resultados relativos ao nível representacional das relações íntimas, verificamos a adequação das escalas de Coerência e Idealização, seja pelo sentido da relação que apresentam entre si, seja pela relação que estabelecem com outras dimensões. Assim, conforme teoricamente antecipado (Crowell \& Owens, 1996), os indivíduos que são claros e precisos no modo como falam sobre a sua relação, sobre si e o outro, apoiando as suas reflexões e avaliações com episódios específicos consistentes, apresentam valores inferiores ao nível da Idealização.

Atendendo, ainda, a estes índices de organização da experiência, sublinharíamos os resultados que sugerem que os sujeitos capazes de organizar coerentemente a sua experiência tendem a representar a relação como promotora de autonomia e desenvolvimento pessoal, em contraste com os indivíduos que idealizam a sua relação e o seu envolvimento pessoal e do companheiro. Uma elevada idealização está também inversamente correlacionada com a qualidade da prestação de cuidados, podendo tal estar associado a um discurso vago e com avaliações pouco sustentadas por memórias específicas. Por seu turno, à representação da prestação de cuidados associa-se a capacidade de manifestar desconforto e solicitar apoio, num balanceamento flexível destes papéis, assumidos como centrais no contexto das relações íntimas (Roisman et al., 2001).

Os resultados apontam para uma associação entre, por um lado, o sentido de autonomia pessoal e envolvimento com o outro e, por outro lado, a capacidade de recorrer ao outro e lhe prestar cuidados em situações de mal-estar, sugerindo que, nesta dialéctica do dar e receber, se consubstancia o equilíbrio entre o desenvolvimento pessoal face ao (e em benefício do) desenvolvimento da relação. Estes dados são encontrados em ambos os domínios de avaliação, sugerindo a convergência das medidas. Similar- 
mente, registam-se valores de correlação significativos quando são consideradas as relações entre o comportamento de Base Segura e a avaliação da Qualidade da Relação, e entre esta e a escala de Desenvolvimento do Indivíduo/Desenvolvimento da Relação. Estes resultados sugerem que quanto mais evidente o balanceamento e integração dos comportamentos de procura e prestação de cuidados, mais complexa e saliente é a relação, permitindo o desenvolvimento pessoal.

Os resultados apresentados indicam, também, que a representação das relações íntimas se associa ao comportamento observado na interacção diádica em diversas dimensões.

Assim, a Idealização encontra-se negativamente associada à experiência de crescimento pessoal no domínio comportamental, ao invés do que sucede com a Coerência, indicando que sujeitos capazes de coerentemente falar sobre a relação e o outro tendem a experienciar níveis mais elevados de desenvolvimento individual e de diferenciação face ao outro. A capacidade de organização reflexiva da experiência está igualmente associada, por um lado, à capacidade de procurar e proporcionar cuidados de modo flexível e, por outro lado, à qualidade global da relação no plano comportamental. Contudo, em contraste com o antecipado, a Idealização da relação não se correlaciona com a Avaliação Global da Qualidade da Relação, podendo tal dever-se à pouca variabilidade desta dimensão na nossa amostra.

Ainda no âmbito da relação entre os domínios representacional e comportamental, mas relativamente a variáveis mais associadas à vinculação, foi encontrada uma correlação positiva entre a escala Equilíbrio entre Desenvolvimento do Indivíduo e Desenvolvimento da Relação e a escala Base Segura, sublinhando a relação entre, por um lado, o reconhecimento das necessidades pessoais e da relação e, por outro, a capacidade de flexivelmente recorrer ao outro e de lhe proporcionar cuidados, se necessário se constituir.

Este estudo preliminar aponta no sentido da pertinência de avaliação da vinculação na idade adulta, no contexto das relações íntimas, pelo recurso à combinação de múltiplos métodos, assumidos como complementares (Crowell et al., 2002). Concomitantemente, aspectos centrais do ponto de vista conceptual recebem aqui suporte empírico, com especial destaque para as dimensões relativas à qualidade da organização da representação e ao modo como estas se associam a comportamentos de vinculação, como procura e prestação de cuidados.

Não obstante a importância destes resultados do ponto de vista empírico e conceptual, este estudo apresenta várias limitações que importa sublinhar. Em primeiro lugar, trata-se de um estudo preliminar, que incide numa amostra reduzida, evidenciando a necessidade de desenvolver e me- 
lhorar, no plano empírico, algumas das escalas, particularmente as relativas à prestação e procura de cuidados. O presente estudo, ao centrar-se na relação entre o modo como o indivíduo representa a relação e, por outro lado, os seus comportamentos na interacção com o outro, não contempla directamente a história desenvolvimental do sujeito ou a história relacional de cada um dos parceiros. No entanto, parece-nos inegável que, não obstante a relação actual se constituir como motor de reconstrução e reelaboração das experiências relacionais anteriores, o modo como o sujeito as organiza assume-se como particularmente saliente no contexto da relação actual (Collins \& Sroufe, 1999), pelo que nos parece relevante uma avaliação mais ampla da organização da vinculação de cada indivíduo. Esta questão será examinada no futuro, com uma amostra maior de participantes, na continuidade do estudo preliminar aqui apresentado.

\section{Referências}

Baldwin, M. W., \& Fehr, B. (1995). On the instability of attachment style ratings. Personal Relationships, 2, 247-261.

Berman, W., Marcus, L. \& Berman, E. (1994). Attachment in marital relations. In M. Sperling \& W. Berman (Eds.), Attachment in adults (pp. 204-231). New York: The Guilford Press.

Bowlby, J. (1969/1984). Attachment and Loss: Attachment. London: Basic Books (2nd. Edition).

Bowlby, J. (1988). A Secure Base: Parent-child Attachment and Healthy Human Development. London: Basic Books.

Bretherton, I. \& Munholland, K. (1999). Internal working models in attachment relationships: a construct revisited. In J. Cassidy \& P. R. Shaver (Eds.), Handbook of attachment: Theory, research, and clinical applications (pp. 355-377). New York: The Guilford Press.

Cassidy, J. (2001). Truth, lies, and intimacy: An attachment perspective. Attachment \& Human Development, 3, 2, 121-155.

Collins, N. L., \& Feeney, B. C. (2000). A safe haven: An attachment theory perspective on support seeking and caregiving in intimate relationships. Journal of Personality and Social Psychology, 78, 1053-1073.

Collins, W. A., Hennighausen, Madsen \& Roisman, G. (1998). Dyadic Coding of Couple Interactions: rating qualitative features of close relationships. Unpublished Coding Manual, Institute of Child Development, University of Minnesota.

Collins, W. A., \& Sroufe, L. A. (1999). Capacity for intimate relationships: A developmental construction. In W. Furman, C. Feiring \& B. B. Brown (Eds.), Contemporary Perspectives on Adolescent Romantic Relationships (pp. 123-147). New York: Cambridge University Press. 
Crittenden, P. (1997). Patterns of attachment and sexual behavior: Risk of dysfunction versus opportunity for creative integration. In L. Atkinson \& K. Zucker, Attachment and Psychopathology (pp. 47-93). New York: The Guilford Press.

Crowell, J., \& Owens, G. (1998). Manual for the Current Relationship Interview and Scoring System, Version 4.

Crowell, J., \& Treboux, D. (2001). Attachment security in adult partnerships. In C. Clulow (Ed.), Adult attachment and couple psychotherapy: the 'secure base' in practice and research (pp. 28-42). Sussex: Brunner-Routledge.

Crowell, J., Treboux, D., Gao, Y., Pan, H., Fyffe, C., \& Waters, E. (2002). Assessing secure base behavior in adulthood: Development of a measure links to adult attachment representations and relations to couples' communication and reports of relationships. Developmental Psychology, 38(5), 679-693.

George, C., Kaplan, N., \& Main, M. (1985). Attachment interview for adults. University of California at Berkeley (Manuscrito não publicado).

Grossmann, K., Grossmann, K., Winter, M., \& Zimmermann, P. (2002). Attachment relationships and appraisal of partnership: from early experience of sensitive support to later relationship representation. In L. Pulkkinen \& A. Caspi (Eds.), Paths to successful development: Personality in the life course. Cambridge: University of Cambridge.

Lima, V., Soares, I., Vieira, F., \& Collins, W. A. (2005). A Intimate Relationship Interview e sistema de cotação, versão 1.0. Universidade do Minho. (Manuscrito não publicado).

Roisman, G. I., Madsen, S. D., Henninghausen, K. H., Sroufe, L. A., \& Collins, W. A. (2001). The coherence of dyadic behaviour across parent-child and romantic relationships as mediated by the internalized representation of experience, Attachment and Human Development, 3(2), 156-172.

Soares, I. (2000). Psicopatologia do desenvolvimento e contexto familiar: Teoria e investigação das relações de vinculação. In I. Soares (Coord.) Psicopatologia do desenvolvimento: Trajectórias (in)adaptativas ao longo da vida (pp. 381-434). Coimbra: Quarteto.

Wampler, P., Shi, Nelson, Kimball, (2003). The adult attachment interview and observed couple interaction: implications for an intergenerational perspective on couple therapy. Family Process, 42, 497-515.

Waters, E., Kondo-Ikemura, K., Posada, G., \& Richters, J. (1991). Learning to love: Mechanisms and milestones. In M. Gunner \& L. A. Sroufe (Eds.), The Minnesota Symposia on Child Psychology (Vol. 23, Self processes in development). pp. 217-255. Hillsdale, NJ: Erlbaum.

Waters, E., \& Cummings, M. (2000). A secure base from which to explore relationships. Child Development, 71, 164-172.

West, M., \& Sheldon-Keller, A. (1994). Patterns of relating: An adult attachment perspective. New York: The Guilford Press. 